\title{
FACTORES QUE IMPIDEN EL LOGRO DE METAS ACADÉMICAS DE UN ESTUDIANTE CENTRALINO DE PREGRADO
}

Cecilia Marcillo, Michelle Blanco, Tania Espinoza, Doris Quinchiguano, Karla Andrade

Universidad Central del Ecuador

Recibido: 12 - septiembre - 2015, aprobado 15 - noviembre - 2015

\section{Resumen}

Los factores que impiden el logro de metas académicas de nuestros estudiantes de la UCE, partió de las inquietudes de qué factores son, de qué manera inciden en los logros académicos, cómo los manejan y si están presentes en todos los estudiantes. Para ello se validó previamente una encuesta que luego se la aplicó a 300 estudiantes aleatoriamente en las distintas facultades de la UCE. Los resultados señalan que en un $28 \%$ de la población encuestada los factores que impiden ellogro de sus metas académicas son el socioeconómico, el familiar, el afectivo y la mala elección de la carrera; el $72 \%$ señala no tener dificultades o haberlas superado. Se concluyó que la mayor dificultad está en los primeros niveles que comprenden edades entre los 18 y 20 años y que de acuerdo al desarrollo psico-emocional y de personalidad del ser humano, la motivación hacia el logro y la obtención de metas se va conquistando conforme su edad avanza.

Palabras clave: logros académicos, desempleo, necesidad, deserción.

\begin{abstract}
The essay about the factors that prevent the achievement of academic goals of our UCE students, started from these concerns: what factors they are?, how they influence the academic achievements?, how they are handled? and if they are present in all students. For this, a survey was previously validated, which then was applied to 300 students randomly in the different faculties of the UCE. The results show that for $28 \%$ of the surveyed population, the factors that prevent the achievement of their academic goals were: the socio-economic, familial, affective and the wrong career choice. The remaining $72 \%$ declared not having difficulties, or having overcome them. It was concluded that the greatest difficulty lies in the first levels that include ages between 18 and 20 years old, and that, accordingly to the psycho-emotional and personality development of the human being, the motivation towards the achievement of goals is conquered as their age advances.
\end{abstract}

Keywords: academic achievements, unemployment, need, desertion. 


\section{Introducción}

En las diferentes facultades de la UCE, existen estudiantes con problemas de distinta naturaleza, ¡Claro, como todo ser humano tienen también problemas!, pero no quiero enfocarlos como "las personas tienen problemas", sino como cuáles son, y qué hacen que muchos abandonen los estudios, o se mantengan con la esperanza de culminarlos pese a toda dificultad, o simplemente los tomen de carrera en carrera hasta culminarlos en alguna de ellas, o que elijan una carrera que luego no era la que realmente querían.

Este contexto me llevó a analizar que existen varios factores que inciden en el logro de metas, y en las decisiones de los estudiantes, y son factores de naturaleza externa como su mismo entorno, sus ingresos, sus amigos, sus experiencias de infantes y adolescentes; así como factores internos como frustraciones, conflictos, carencias, duelos, pérdidas, que les causan infelicidad, insatisfacción, e incluso en algunos despiertan ideas suicidas y lo peor, en uno u otro caso las ejecutan.

Estos factores externos e internos son inherentes a ellos pues forman parte de su personalidad y desarrollo psicológico, si alguno de ellos no es satisfecho de manera positiva causa conflicto o frustración y esto a su vez acarrea baja autoestima, desmotivación, desorientación y posiblemente el logro de metas sin satisfacción total; es decir, alcanzar un título pero no haber desarrollado el total de su potencial cognitivo.

Esta podría ser una de las causas por la que en algunos estudiantes de semestres superiores se observan competencias bajas en cuanto a resumir, preparar ensayos, revisar bibliografía y aplicar en las diferentes asignaturas.

Los factores que impiden que un estudiante logre sus metas académicas se observan sobre todo en los tres niveles primeros y en edades entre los 18 y 20 años y uno que otro entre los 21 a 26 años.

La vida de muchos de ellos no solo se centra en estudiar; algunos deben trabajar para aportar en casa aunque esto les cueste dejar sus estudios, otros cuidan de sus hermanos menores y estudian, otros ven la forma de ahorrar porque no alcanza el dinero en la familia, muchos, sobre todo aquellos que vienen de otras provincias viven con lo justo; y hay otra población que estudia lo que no le gusta porque por ley están donde su puntaje los ubicó, o porque no pueden acceder a universidades particulares por lo costoso que resulta, o que iniciaron una carrera pensando en los réditos económicos y al ver la competencia sienten frustración y desesperanza.

Sin embargo, son los estudiantes de nivel socioeconómico bajo, los que verdaderamente presentan estas dificultades y aunque constituya el $28 \%$ de esta investigación, son representantes de los problemas que debemos ayudar a subsanar.

La pregunta que surge es, si esto es real, ¿qué pasa con nuestros chicos, qué estamos haciendo para apoyarlos?, qué hace la Universidad, qué hacemos los docentes; será que la falta de apoyo u orientación aporta a este sentir, quizá esto tenga que ver con la producción académica, quizá podrá influir en algo en el prestigio de nuestra gloriosa Universidad o es simplemente un hecho que pasa. 
Existen muchos estudios en relación a la deserción académica, se debe seguir insistiendo en aplicar las sugerencias que derivan de los mismos hasta lograr disminuir estas estadísticas.

\section{Métodos y Materiales}

La presente investigación quiso conocer qué factores impiden a los estudiantes el logro de sus metas académicas, para eso se estableció una encuesta que fue previamente validada por 50 estudiantes encuestados, de esta se desprendió la encuesta definitiva con la que se trabajó.

Una vez establecida la misma, se aplicó aleatoriamente a 300 estudiantes de diferentes facultades de la UCE, de ambos sexos, de diferentes semestres y de edades entre los 18 a 26 años.

\section{Resultados}

Las preguntas planteadas hicieron referencia a si se siente satisfecho o no el estudiante con su vida, a los problemas que los aquejan, a cómo los afrontan, y qué necesitan para alcanzar sus metas; los resultados obtenidos fueron:

- De los 300 estudiantes encuestados, el 52\% corresponde al género femenino y el 47,5\% al género masculino.

- Las edades fluctúan entre los 18 y 26 años.

- Los estudiantes que participaron fueron de las diferentes facultades de la UCE según muestra el cuadro, donde Psicología, Comunicación Social, Filosofía y Jurisprudencia tuvieron mayor representatividad.

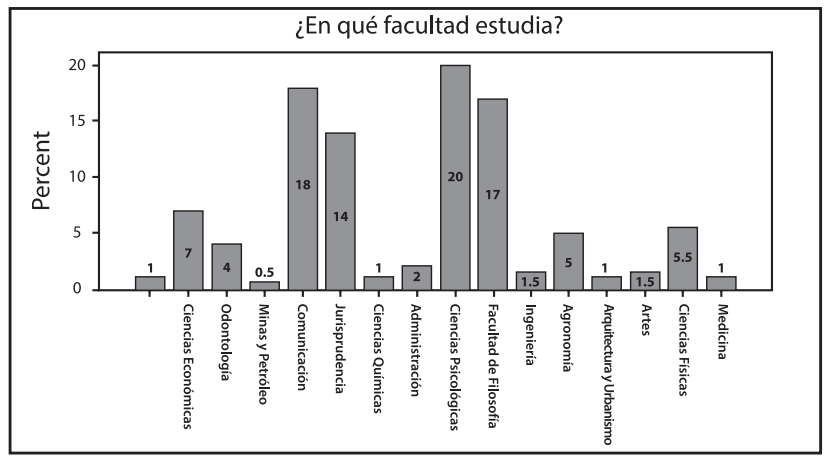

- La pregunta sobre la satisfacción o no de su vida actual, señala que el $71 \%$ está satisfecho con su vida por contar con el apoyo económico y afectivo de sus padres, de su pareja; algunos tienen empleo de medio tiempo, otros porque ya superaron los primeros niveles y están más empoderados de sus actos. El 28\% de estudiantes respondieron que su vida es poco satisfactoria, incompleta y un $1 \%$ que su vida es deprimente. 


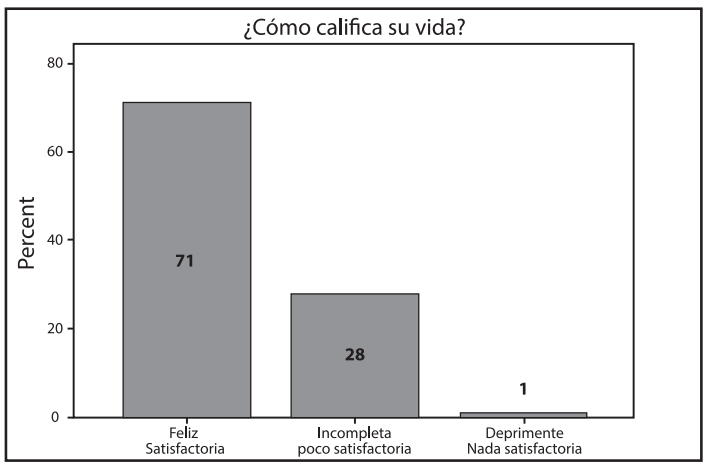

- Al consultarles qué situación les urge solucionar las respuestas en orden de representatividad fueron las siguientes:

- Familiar con el 14,67\%, donde señalan carencia afectiva, desorganización familiar, malas relaciones con sus padres, desunión familiar, abandono, nadie los escucha.

- Económico con el 12\%, donde señalan la necesidad de aportar económicamente en su casa, dejar de ser "carga", valerse por sí mismos para salir de su hogar.

- De pareja, con el 8,66\% donde manifiestan no tener pareja, tenerla y no entenderse, no ser correspondido, ruptura y abandono.

- Académico con el 3,33\%, donde manifiestan no sentirse a gusto con su carrera por mala elección, no sentirse a gusto porque la carrera y los docentes no cumplen sus expectativas, porque algunos reciben mal trato de sus profesores, por no entender algunas asignaturas y porque siguen la carrera por el puntaje obtenido en la SENESCYT.

- El 19,33\% respondió otros factores, entre ellos está no tener actitud ante las dificultades, no conformarse con su estado actual, no saber qué es lo que realmente quieren, no entienden su entorno, se sienten solos, tienen responsabilidades fuertes como las de ser madres precoces y les falta tiempo para cuidar a sus hijos y un papá que las apoye en su cuidado y manutención.

- Un $42 \%$ responde que nada por ahora, o manifiestan necesidades como alcanzar el título, viajar, tener un auto u obtener cosas materiales por sus propios medios.

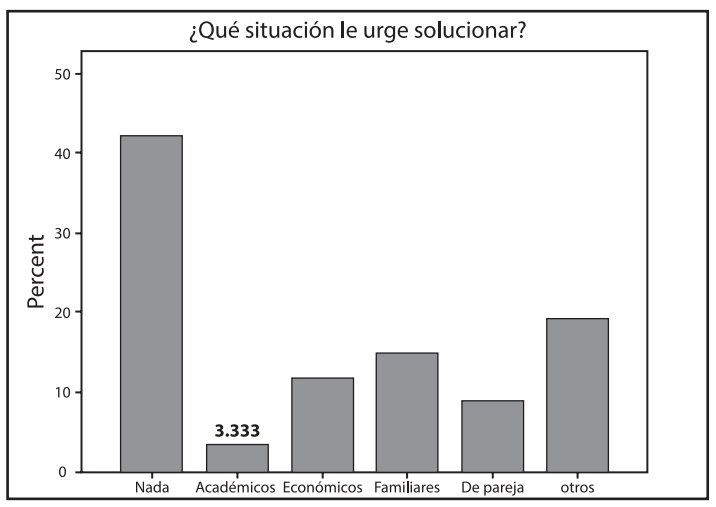


- Al preguntar sobre lo que les hace falta para sentirse mejor consigo mismo y con los demás, las respuestas difieren en el orden entre el factor familiar y el económico en relación a los resultados de la pregunta sobre lo que les urge solucionar. El factor económico/empleo ocupa el primer lugar y el factor familiar el segundo, los demás factores siguen el mismo orden.

Podemos notar sin embargo que el 52\% requiere trabajo y aumenta la necesidad de un mejor trato por parte de las autoridades y profesores de la universidad a un $13.5 \%$, lo que indica que pese a que el $42 \%$ no tiene problemas relevantes, sí se adhieren a la necesidad de trabajar y a un mejor trato por parte de los profesores y autoridades.

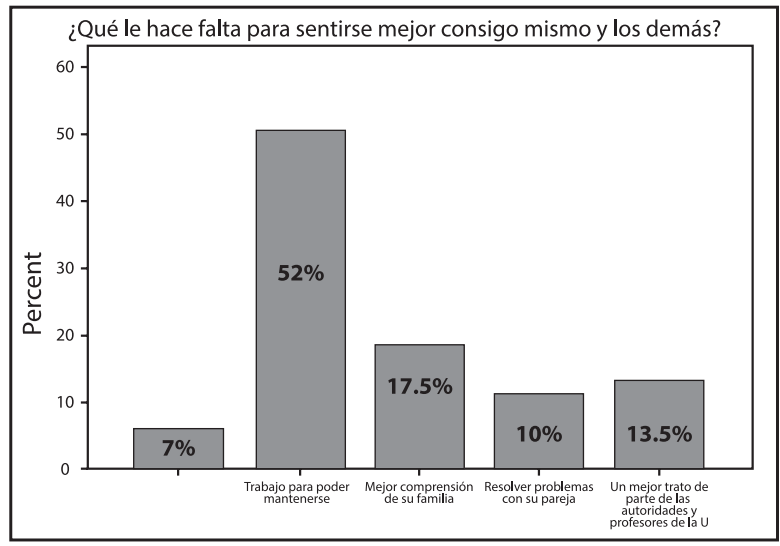

- En cuanto al afrontamiento de sus problemas, un 56,5\% razona y soluciona el problema de manera adecuada. El 26\% pide asesoramiento, el 5\% no encuentra la solución y un 11,5\% considera que tienen una solución que solo ellos conocen (manifiestan querer escapar de su realidad y olvidarse de todo, abandonar los estudios, irse de esta vida). El 1\% no da respuesta.

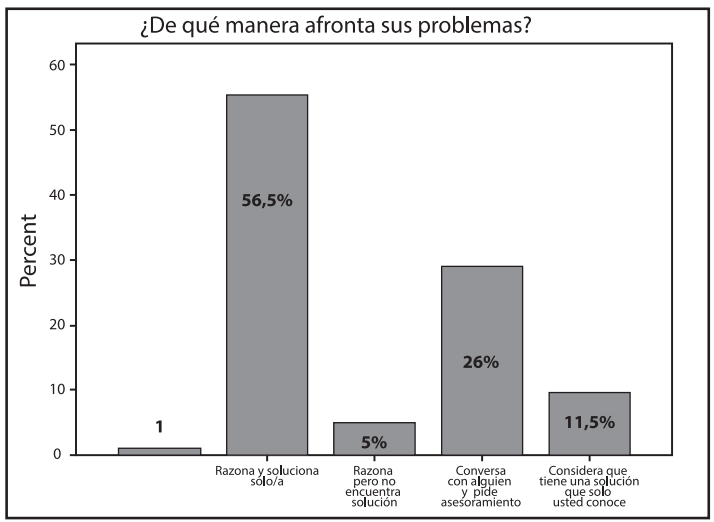

- Las respuestas en relación a lo que prefieren cuando tienen problemas fue: ser escuchados en un 39\%, ser abrazados en un 18\%, ser aconsejados en un $17,5 \%$, en un $14 \%$ precisa otras cosas como ir a vivir solos, tener muchos regalos. El $8,5 \%$ dice todos y un 3\% no responde. 


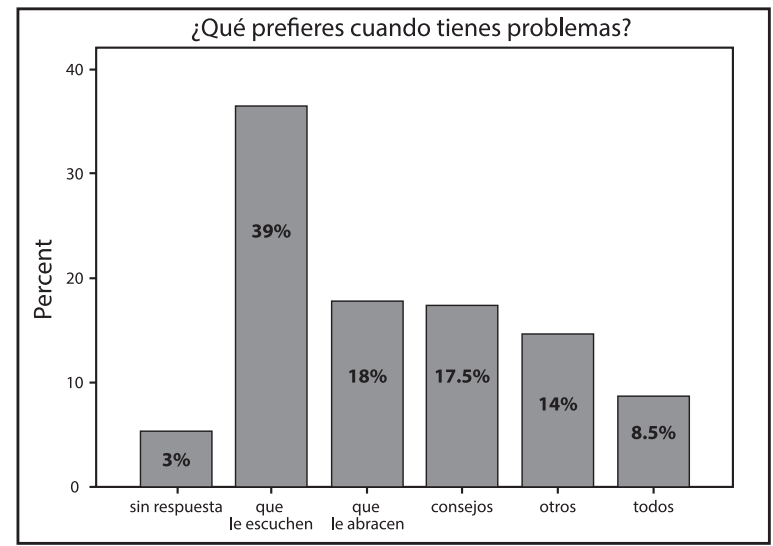

\section{Análisis y Discusión}

En el Ecuador, estudios realizados por la Politécnica Nacional, la Escuela Politécnica del Ejército, extensión Latacunga, la UCE, la PUCE, coinciden en señalar que aproximadamente el $73.11 \%$ de la población estudiantil de pregrado deserta de sus estudios por factores socioeconómicos, de escogitamiento de carrera, de superpoblación, personales, de embarazo precoz, costos elevados, entre otros.

Un análisis de estudios sobre rendimiento de los programas académicos universitarios en Colombia, cuya autora es la Dra. Adelaida Salcedo E., publicado en la Revista Academia y Virtualidad de la Universidad Militar Nueva Granada, indica algunos factores que inciden en la deserción universitaria en Colombia, hace referencia a factores como la falta de planificación y programación de la universidad, el sistema educativo nacional, el factor socioeconómico adverso, así como a factores externos e internos, políticas currículos, competencias establecidas por la misma universidad.

De la misma forma, otro estudio realizado en el Perú por Juan Tonconi Quispe en la Facultad de Ingeniería económica de la UNA-PUNO, 2009, en relación al rendimiento académico y deserción estudiantil, señala que entre los factores que marcan la deserción y el bajo rendimiento es el económico, seguido del familiar.

El presente estudio, no se enfoca en la deserción estudiantil propiamente, sino en los factores que dificultan el logro de metas académicas y que día a día deben sortear los estudiantes; sin embargo, coincidimos con otros estudios, que el factor socioeconómico es relevante e incide en las decisiones que toman nuestros estudiantes y que posiblemente a muchos los lleve a desertar, sin embargo, el factor familiar es también considerable e incide con fuerza en el logro de metas académicas. 


\section{Conclusiones}

- En los dos primeros semestres y en edades entre los 18 a 20 años, los resultados señalan inestabilidad emocional, baja autoestima, desorientación; el porcentaje corresponde al $28 \%$ de este estudio, incluido uno que otro de edad más avanzada, lo que indica que en los primeros niveles se deben fortalecer los factores que permitirán lograr sus metas académicas a futuro, ya que de acuerdo desarrollo psico-emocional y de personalidad del ser humano, la motivación hacia el logro y la obtención de metas se va conquistando conforme su edad avanza, es decir, a mayor edad cronológica, mayor madurez emocional. Aunque esto no es una regla sine qua non, puede ser asimilación, adaptación y conformidad en algunos casos.

- Cuando un estudiante rebasa la barrera de sus problemas, se adapta, lucha por sus ideales pese a toda dificultad, la consecución de metas es más próxima.

- Seguir una carrera por sugerencia de los padres, por falta de recursos, por puntajes obtenidos, sin ser la de sus sueños, resulta frustrante para todo ser humano. Podemos equivocarnos en tantas cosas, pero es importante prepararnos para servir desde nuestra vocación.

- Lamentablemente los resultados de la rendición de pruebas previo al ingreso a las universidades, no es tan real, muchos estudiantes ingresan a donde pueden para después cambiarse de facultad o carrera, en ello pierden tiempo y recursos, sobre todo las familias que no lo poseen.

\section{Recomendaciones}

- Las universidades a través de sus autoridades deben sugerir al Gobierno, al Ministerio de Educación, que se haga una verdadera orientación vocacional a los estudiantes de los últimos años para evitar el cambio de carreras o la deserción en la universidad.

Además, que se revea el sistema de pruebas, ya que muchos estudiantes no solo de la Central, están estudiando carreras que no les gusta, pero que deben culminar.

- Cada Facultad debe tener su propio Departamento de Consejería Estudiantil para brindar apoyo psicológico a tiempo, de tal suerte que al ser una universidad gratuita, aprovechen este recurso y no se queden con sus problemas por no tener dinero para consultas particulares.

- Mantener las becas para los buenos estudiantes, e incentivar a través de campañas y socializar a través de las redes que todo estudiante que tenga un buen promedio será beneficiario de una beca. No todos conocen esto.

La vida que nos trazamos, los logros que alcanzamos, en gran parte son producto de nuestro propio esfuerzo, de nuestra actitud y de las decisiones que tomamos. Muchas veces pese a que hemos madurado cronológicamente, nuestra madurez emocional nos traiciona y nos equivocamos en las decisiones que tomamos, pero el reto a vencer es superarlo, pues somos el resultado de las mismas. La vida no resulta para todos tan solvente, el entorno familiar, social, laboral, a veces nos 
juega una mala pasada y nos presiona, y pese a todo debemos tomar decisiones sabiendo que el dolor de hoy será sin duda el gozo del mañana.

\section{Bibliografía}

Centro de Microdatos. Estudio sobre las causas de la deserción universitaria. Chile Departamento de Economía. Universidad de Chile. Agosto, 2008.

http://www.oei.es/pdf2/causas-desercion-universitaria-chile.pdf

Collantes, Iván (2012). Deserción de estudiantes universitarios en la ESPE-Latacunga. Ecuador

http:/ / espeldesercion.blogspot.com/\#!

Montenegro, Carlos, Taco, M. Belén (2012). Repitencia y deserción de los estudiantes de pregrado de las facultades de Arquitectura y Administración. Tesis previa a la obtención del título de economista. UC del Ecuador.

http://www.dspace.uce.edu.ec/bitstream/25000/957/1/T-UCE-0005-155.pdf

Pontificia Universidad Católica del Ecuador (2011). Estudio sobre la deserción estudiantil de la PUCE. Repositorio digital

http:/ / repositorio.puce.edu.ec/handle/22000/3454

Salcedo, Adelaida (2000). Deserción universitaria en Colombia. Revista Académica y Virtualidad, Universidad militar Nueva Granada. Colombia. http://www.alfaguia.org/alfaguia/files/1319043663_03.pdf

Tonconi Q., Juan (2010). Factores que influyen en el rendimiento académico y deserción de los estudiantes de la Facultad de Ingeniería Económica de la UA-PUNO, período 2009. Revista académica semestral, vol. 2, No 11 . Perú.

http://www.eumed.net/rev/ced/11/jtq.htm 